\title{
USO DE DROGAS PISCOTRÓPICAS POR ADOLESCENTES DE ESCOLAS PÚBLICAS
}

\author{
Bruno Miranda Pereira ${ }^{1}$, Karolyne Araujo Resende ${ }^{2}$, Cecília Godoi Campos ${ }^{3}$, Sebastião Júnior Henrique Duarte ${ }^{4}$, Ricardo
} Bezerra Cavalcante $^{5}$, Richardson Miranda Machado ${ }^{6}$

\begin{abstract}
${ }^{1}$ Discente do Ensino Médio. Escola Estadual Padre Matias Lobato. Divinópolis, MG, Brasil.
${ }^{2}$ Enfermeira. Residente em Saúde do Adolescente. Universidade Federal de São João del-Rei. Divinópolis, MG, Brasil.

${ }^{3}$ Enfermeira. Especialista e Mestre em Saúde Mental. Coordenadora do Núcleo de Atenção Primária à Saúde da Secretaria de Estado da Saúde de Minas Gerais / Regional Oeste. Divinópolis, MG, Brasil.

${ }^{4}$ Enfermeiro. Pós-Doutorando em Enfermagem em Saúde Coletiva. Docente da Universidade Federal de Mato Grosso do Sul. Divinópolis, MG, Brasil.

${ }^{5}$ Enfermeiro. Mestre em Enfermagem. Doutor e Pós-Doutor em Ciência da Informação. Docente da Universidade Federal de São João del-Rei. Divinópolis, MG, Brasil.

${ }^{6}$ Enfermeiro. Mestre em Enfermagem. Doutor em Psiquiatria. Docente da Universidade Federal de São João del-Rei. Divinópolis, MG, Brasil.
\end{abstract}

RESUMO: Objetivou-se identificar a associação entre características sociodemográficas e o uso de álcool e outras drogas por adolescentes de escolas públicas municipais. Pesquisa exploratória, realizada no primeiro semestre de 2014. Participaram 480 escolares em Divinópolis, Minas Gerais, selecionados por amostra não-probabilística, que responderam individualmente a um questionário elaborado pelos autores e ao Drug Use Screening Inventory. A análise ocorreu pela estatística descritiva e bivariada. Os resultados apontaram a predominância do sexo masculino (51,9\%), com renda familiar de 1 a 2 salários mínimos (52,7\%). Usam analgésicos (43,9\%), álcool (39,5\%) e tabaco $(9,7 \%)$. Quanto às variáveis que favoreciam o uso de drogas, a escassez de lazer e recreação apresentaram a maior frequência entre problemas relatados. Os achados indicam a necessidade da implementação de políticas voltadas à saúde integral dos adolescentes, em especial às atividades culturais, esportivas, laborais e lúdicas, como medidas na prevenção e enfrentamento ao uso e abuso de álcool e outras drogas.

DESCRITORES: Atenção primária à saúde; Detecção do abuso de substâncias; Comportamento do adolescente; Transtornos mentais.

\section{PSYCHOTROPIC DRUG USE AMONG TEENAGE PUBLIC SCHOOL STUDENTS}

ABSTRACT: The aim of this study was to identify the association between socio-demographic characteristics and use of alcohol and other drugs by teenage public school students. This was an exploratory study, conducted in the first half of 2014. A total of 480 public school students from the municipality of Divinópolis, in the Brazilian state of Minas Gerais, were selected to participate in the study by non-probabilistic sampling. The participants responded individually to a questionnaire prepared by the authors and the Drug Use Screening Inventory. Analysis was by descriptive and bivariate statistics. The results showed a predominance of male users $(51.9 \%)$, with family income $1-2$ times the minimum wage $(52.7 \%)$. Analgesics $(43.9 \%)$ were the primary drugs used, followed by alcohol (39.5\%) and tobacco $(9.7 \%)$. As for variables that favored drug use, lack of leisure and recreation activities had the highest frequency of reported problems. The findings of this study indicate the need to implement policies aimed at comprehensive health care for adolescents, especially cultural, sporting, leisure and professional training activities, as well as measures to prevent the use and abuse of alcohol and other drugs.

DESCRIPTORS: Primary health care; Substance abuse detection; Adolescent behavior; Mental disorders.

\section{USO DE DROGAS PISCOTRÓPICAS POR ADOLESCENTES DE ESCUELAS PÚBLICAS}

RESUMEN: La finalidad del estudio fue identificar la asociación entre características sociales y demográficas y el uso de alcohol y otras drogas por adolescentes de escuelas públicas municipales. Investigación exploratoria realizada en el primero semestre de 2014. Participaron 480 escolares en Divinópolis, Minas Gerais, seleccionados por muestra no probabilística, que contestaron individualmente a un cuestionario elaborado por los autores y al Drug Use ScreeningInventory. El análisis ocurrió por la estadística descriptiva y bivariada. Los resultados apuntaron la predominancia del sexo masculino $(51,9 \%)$, con renta familiar de 1 a 2 salarios mínimos (52,7\%). Usan analgésicos (43,9\%), alcohol $(39,5 \%)$ y tabaco $(9,7 \%)$. Cuanto a las variables que favorecían el uso de drogas, la escasez de ocio y recreación presentaron la mayor frecuencia entre problemas relatados. Los resultados indican la necesidad de la implementación de políticas para la salud integral de los adolescentes, en especial las actividades culturales, esportivas, laborales y lúdicas, como medidas en la prevención y afrontamiento al uso y abuso de alcohol y otras drogas.

DESCRIPTORES: Atención primaria a la salud; Detección del abuso de substancias; Comportamiento del adolescente; Trastornos mentales. 


\section{INTRODUÇÃO}

$\mathrm{Na}$ atualidade, o uso abusivo de álcool e/ ou drogas é caracterizado como um fenômeno social que representa um grave problema à saúde pública mundial, fato que tem despertado a atenção das autoridades de saúde e sanitárias de diversos países. Possui como principal característica, segundo a Organização Mundial de Saúde (OMS), o estado físico e psíquico alterado, resultante da ingestão de substâncias químicas, com o surgimento de reações comportamentais que incluem compulsão pela ingestão contínua ou periódica da substância. Sendo a principal finalidade de o seu consumo experimentar os efeitos físicos causados pela droga e evitar o desconforto ocasionado pela sua falta ${ }^{(1)}$.

O uso e abuso de álcool e/ou drogas é tido como um dos dez comportamentos de maior risco à saúde, e constitui causa da morte de 1,8 milhões de pessoas no mundo, destas $5 \%$ são jovens entre os 15 e 29 anos de idade. Os dados revelam que as pessoas ainda estão longe de ter uma relação equilibrada com as substâncias químicas ${ }^{(2-3)}$.

Um levantamento nacional evidenciou que $12,3 \%$ das pessoas, com idades entre 12 e 65 anos, possuem algum tipo de dependência química, com prevalência de $17,1 \%$ entre a população masculina e $5,7 \%$ na população feminina. Outros estudos realizados no Brasil e no exterior demonstraram que, quanto mais cedo se começa a ingerir álcool e/ou drogas, maior o risco de se tornar dependente e de se ter problemas clínicos e sequelas. Também há o aumento da probabilidade de ocorrerem internações, acidentes, brigas, assassinatos, suicídios e de fazer sexo sem proteção ${ }^{(4-5)}$.

Dados do último Inquérito Nacional sobre Dependência realizado no Brasil demonstraram que $55,6 \%$ da população maior de 18 anos havia consumido álcool com frequência nos últimos 12 meses. Também observaram-se aumentos dramáticos nos índices de consumo de álcool entre adolescentes, com 35\% em 1999 consumindo bebidas alcoólicas, enquanto no ano de 2001 esse índice subiu para $48 \%$. Problemas relacionados ao consumo de álcool foram relatados por $4 \%$ e $10 \%$ dos entrevistados nas faixas etárias de 12-17 e 18-25 anos respectivamente ${ }^{(6-9)}$.

Autores ressaltam que os primeiros contatos com o álcool e/ou drogas pelos adolescentes, na maioria dos casos, estão associados aos instintos naturais de um ser "em fase peculiar de desenvolvimento: curiosidade, imitação, autoafirmação". Há também outras causas relacionadas a processos psicológicos autodestrutivos de origem individual, familiar ou social, como a vontade de transgredir regras, a revolta contra todos, a opressão social ou econômica ou até mesmo deficiências mentais" ${ }^{\prime(7,9)}$.

Dados da Organização das Nações Unidas (ONU) indicam que a maioria dos jovens se envolve com o álcool e/ou drogas por falta de informação. Assim, na busca de sua própria identidade, muitas vezes adotam comportamentos inadequados, influenciados por amigos ou conhecidos e acabam por fazer uso de substâncias sem conhecerem seus prejuízos e tornam-se dependentes químicos.

Tomando a relevância da temática e na intenção de contribuir com a produção do conhecimento a respeito dos malefícios provocados pelo uso do álcool e/ou drogas por adolescentes, este estudo teve por objetivo identificar a associação entre características sociodemográficas e o uso de álcool e outras drogas por adolescentes de escolas públicas municipais.

\section{METODOLOGIA}

Trata-se de um estudo exploratório e transversal, realizado com adolescentes de duas escolas municipais localizadas na cidade de Divinópolis, estado de Minas Gerais, consideradas pela Secretaria Municipal de Educação como as maiores do município, com cerca de 600 alunos matriculados, desses 500 são adolescentes. Ambas as escolas são responsáveis pelo ensino básico de crianças de 6 a 11 anos de idade e pelo ensino fundamental de adolescentes de 12 a 18 anos. Os alunos em sua maioria são provenientes de bairros localizados na periferia da cidade, áreas desprovidas de infraestrutura básica e de lazer.

Foram adotados como critérios de inclusão: a) aceitar participar do estudo, b) preencher e assinar o Termo de Assentimento Livre e Esclarecido (TALE); c) ter a assinatura de um responsável legal no Termo de Consentimento Livre e Esclarecido (TCLE) e, d) ser aluno de uma das duas escolas há, no mínimo, seis meses. E como critérios de exclusão: preenchimento errôneo ou incompleto do TALE, do TCLE ou dos questionários utilizados para o estudo.

Foram convidados para participar da pesquisa todos os $500(100 \%)$ adolescentes estudantes das duas escolas. A amostra final foi constituída por $480(96 \%)$ adolescentes devido a questionários não respondidos ou incompletos. 
Para a coleta dos dados, foram utilizados dois questionários, um elaborado pelos autores da pesquisa para abordar os dados sociodemográficos (sexo, idade, religião, raça, presença parental e renda familiar) e o outro denominado Drug Use Screening Inventory (DUSI), desenvolvido nos Estados Unidos e adaptado à população brasileira (Inventário de Triagem do Uso de Drogas) ${ }^{(10)}$. Essa versão é direcionada especialmente à população adolescente, de domínio público e disponibilizada gratuitamente.

Ressalta-se que o DUSI é composto por uma tabela inicial que aborda a frequência de consumo de 13 classes de substâncias psicoativas, seguida por 149 questões divididas em 10 áreas, fornecendo um perfil da intensidade de problemas em relação ao uso de substância, comportamento, saúde, transtornos psiquiátricos, sociabilidade, sistema familiar, escola, trabalho, relacionamento com amigos e lazer/recreação. As questões foram respondidas com "sim" ou "não", sendo que as respostas afirmativas equivaleram à presença de problemas. Além das 10 áreas mencionadas, o DUSI possui uma "escala de mentira", composta por 10 questões (uma no final de cada área) que foram utilizadas com a finalidade de checar a existência de possíveis questionários inválidos ${ }^{(10)}$.

Os dados foram coletados no decorrer do primeiro se $\neg$ mestre do ano de 2014, após a aprovação pelo Comitê de Ética e Pesquisa envolvendo seres humanos do Hospital São João de Deus, Parecer $n^{\circ} 59 / 2013$. Os instrumentos foram respondidos individualmente por alunos dos turnos matutino e vespertino, em local privativo e somente na presença de um dos pesquisadores. A média de tempo para resposta ao questionário foi de 30 a 40 minutos. Cada etapa da pesquisa foi descrita aos respondentes de forma simples e com uso de vocabulário de fácil entendimento. Os adolescentes foram orientados a após terminarem de responder os questionários, depositá-los em uma urna, preservando-se o anonimato e a identificação de cada participante.

Os dados obtidos foram digitados em planilhas do aplicativo Excel, através da técnica de dupla entrada. Posteriormente foram exportados para o software Statiscal Package for Social Sciences (SPSS) versão 15.0, por meio do qual se determinou a significância estatística, calculou-se o qui-quadrado de Person (x2) e quantificou-se a associação entre as variáveis dos participantes. Utilizou-se o modelo de regressão logística para a análise do uso de drogas, através do cálculo do Odds Ratios (OR) bruto e Odds Ratios ajustado para todas as co-variáveis. As evidências de associação no nível de 0,05 de significância foram observadas se o valor 1 não estivesse contido nos intervalos de confiança. Na estimativa do OR ajustado, foi utilizado como controle as variáveis grau, convivência e relacionamento. Por fim, o nível de problemas pelo uso de drogas foi estabelecido a partir dos escores gerados pelo DUSI, mediante os quais quatro índices puderam ser calculados: 1) densidade absoluta de problemas, que indicou a intensidade de problemas em cada área isoladamente; 2 ) densidade relativa de problemas, que indicou a contribuição percentual de cada área no total de problemas; 3) densidade global de problemas, que indicou a intensidade geral dos problemas; e 4) escala de mentira, que refletiu a confiabilidade das respostas fornecidas ${ }^{(10)}$.

\section{RESULTADOS}

$\mathrm{Na}$ caracterização dos participantes, chama atenção o elevado número de adolescentes que referiram uso de drogas, independente do sexo, idade, religião, raça, entre outras variáveis, conforme ilustrado na Tabela 1.

$\mathrm{Na}$ análise do total da amostra, houve distribuição semelhante em relação ao sexo, com 51,9\% adolescentes do sexo masculino e $48,1 \%$ do sexo feminino. Entre estudantes do sexo masculino, $50,5 \%$ assinalaram uso de drogas e do sexo feminino $44,9 \%$, com tendência estatística de $p=0,011$. A média de idade foi de 13,03 anos ( $D P=0,99$ anos) e apresentou maior consumo de drogas entre os estudantes de 16 a 18 anos $(p>0,005)$. No que se refere à religião, os participantes com maior porcentagem de uso de drogas $(p=0,000, X 2=30,01)$ denominaram-se católicos $(47,7 \%)$.

Quanto à raça, a maior porcentagem de uso de drogas foi observada entre os adolescentes da raça amarela/oriental $(68,5 \%)$, seguidos pelos que se declararam negros $(38,7 \%)$. No que tange à presença parental, o maior uso de drogas ocorreu com os adolescentes que relataram conviver somente com o pai $(76,4 \%)$, seguidos pelos que relataram não conviver nem com o pai e nem com a mãe $(71,4 \%)$ e pelos que convivem só com a mãe $(59 \%)(p<0,005)$. A renda familiar dos $50,1 \%$ estudantes que haviam consumido drogas estava entre 1 a 2 salários mínimos, enquanto outros $29,2 \%$ relataram renda familiar de 3 a 4 salários $(\mathrm{p}>0,005)$.

As drogas relatadas pelos adolescentes foram: os analgésicos $(43,9 \%)$, o álcool $(39,5 \%)$, seguidos pelo tabaco $(9,7 \%)$, mas também se encontrou 
Tabela 1 - Regressão logística para o uso de drogas, segundo informações sóciodemográficas de 480 estudantes de escolas públicas. Divinópolis, Minas Gerais, Brasil, 2014

\begin{tabular}{|c|c|c|c|c|c|c|c|c|}
\hline \multirow{3}{*}{ Variáveis } & \multirow{2}{*}{\multicolumn{2}{|c|}{$\begin{array}{c}\text { Caracterização } \\
\text { Adolescentes }\end{array}$}} & \multicolumn{4}{|c|}{ Uso de Drogas } & \multicolumn{2}{|c|}{ Odds Ratio } \\
\hline & & & \multicolumn{2}{|c|}{ Não } & \multicolumn{2}{|c|}{ Sim } & \multirow{2}{*}{$\begin{array}{c}\text { OR bruto IC } \\
(95 \%)\end{array}$} & \multirow{2}{*}{$\begin{array}{c}\text { OR ajustado } \\
\text { IC }(95 \%) \\
\end{array}$} \\
\hline & $\mathrm{n}$ & $\%$ & $\mathrm{n}$ & $\%$ & $\mathrm{n}$ & $\%$ & & \\
\hline \multicolumn{9}{|l|}{ Sexo } \\
\hline Feminino & 227 & 48,1 & 125 & 55,1 & 102 & 44,9 & - & - \\
\hline Masculino & 253 & 51,9 & 125 & 49,5 & 128 & 50,5 & $\begin{array}{c}1.531 \\
(1.016 ; 2.30)\end{array}$ & $\begin{array}{c}1.299 \\
(0.835 ; 2.02)\end{array}$ \\
\hline \multicolumn{9}{|l|}{ Idade } \\
\hline 10 a 12 anos & 177 & 36,8 & 107 & 60,5 & 70 & 39,5 & - & - \\
\hline 13 a 15 anos & 280 & 58,3 & 142 & 50,8 & 138 & 49,2 & - & - \\
\hline 16 a 18 anos & 23 & 4,9 & 1 & 4,4 & 22 & 95,6 & $\begin{array}{c}3523 \\
(2.024 ; 311)\end{array}$ & $\begin{array}{c}2.685 \\
(1.324 ; 302)\end{array}$ \\
\hline \multicolumn{9}{|l|}{ Religião } \\
\hline Católico & 354 & 73,7 & 185 & 52,3 & 169 & 47,7 & $\begin{array}{c}1.736 \\
(1.242 ; 15.2) \\
\end{array}$ & $\begin{array}{c}1.826 \\
(1.932 ; 3.33) \\
\end{array}$ \\
\hline Evangélico & 77 & 16,1 & 42 & 54,6 & 35 & 45,4 & - & - \\
\hline Espírita & 10 & 2,1 & 6 & 60 & 4 & 40 & - & - \\
\hline Outros & 39 & 8,1 & 17 & 43,6 & 22 & 56,4 & - & - \\
\hline \multicolumn{9}{|l|}{ Raça } \\
\hline Branca & 196 & 40,8 & 120 & 61,3 & 76 & 32,2 & - & - \\
\hline Negra & 31 & 6,5 & 21 & 67,8 & 10 & 38,7 & $\begin{array}{c}2.112 \\
(1.32 ; 3.37) \\
\end{array}$ & $\begin{array}{c}2.169 \\
(1.318 ; 3.57) \\
\end{array}$ \\
\hline Mulata/Morena & 72 & 15 & 60 & 83,4 & 12 & 16,6 & - & - \\
\hline Amarela/Oriental & 181 & 37,7 & 57 & 31,5 & 124 & 68,5 & $\begin{array}{c}2.413 \\
(1.347 ; 4.32) \\
\end{array}$ & $\begin{array}{c}2.193 \\
(1.171 ; 4.10) \\
\end{array}$ \\
\hline \multicolumn{9}{|l|}{ Presença Parental } \\
\hline Pai e Mãe & 356 & 74,2 & 203 & 57,1 & 153 & 42,9 & - & - \\
\hline Apenas a Mãe & 100 & 20,8 & 41 & 41 & 59 & 59 & $\begin{array}{c}1.488 \\
(0.731 ; 3.02) \\
\end{array}$ & $\begin{array}{c}1.432 \\
(0.662 ; 3.09) \\
\end{array}$ \\
\hline Apenas o Pai & 17 & 3,5 & 4 & 23,6 & 13 & 76,4 & $\begin{array}{c}2.481 \\
(0.408 ; 15.0) \\
\end{array}$ & $\begin{array}{c}1.01 \\
(0.158 ; 6.45) \\
\end{array}$ \\
\hline $\begin{array}{l}\text { Nem Mãe e Nem } \\
\text { Pai }\end{array}$ & 7 & 1,5 & 2 & 28,6 & 5 & 71,4 & $\begin{array}{c}1.815 \\
(1.011 ; 3.25) \\
\end{array}$ & $\begin{array}{c}1.96 \\
(1.041 ; 3.68) \\
\end{array}$ \\
\hline \multicolumn{9}{|l|}{ Renda Familiar } \\
\hline $\begin{array}{l}1 \text { a } 2 \text { salários } \\
\text { mínimos }\end{array}$ & 253 & 52,7 & 126 & 49,9 & 127 & 70,8 & $\begin{array}{c}4.827 \\
(1.169 ; 19.9) \\
\end{array}$ & $\begin{array}{c}2.766 \\
(0.618 ; 12.3) \\
\end{array}$ \\
\hline 3 a 4 salários & 158 & 32,9 & 46 & 29,2 & 112 & 50,1 & $\begin{array}{c}3.851 \\
(2.107 ; 703) \\
\end{array}$ & $\begin{array}{c}2.825 \\
(1.136 ; 7.02) \\
\end{array}$ \\
\hline 5 a 6 salários & 63 & 13,1 & 40 & 63,5 & 23 & 36,5 & - & - \\
\hline 7 ou mais salários & 6 & 1,3 & 4 & 66,7 & 2 & 33,3 & - & - \\
\hline
\end{tabular}

o uso de inalantes/solventes $(3,9 \%)$, maconha $(3,9 \%)$, anfetaminas $(3,7 \%)$, tranquilizantes $(3,7 \%)$ e, em menor porcentagem, cocaína, anabólicos, opiáceos e alucinógenos. Ressalta-se que a frequência do uso ocorreu de modo experimental, tendo $93 \%$ dos adolescentes relatado que tinham consumido drogas com a frequência de uma a duas vezes no último mês. Entre os principais fatores identificados para o uso de drogas estavam a curiosidade, não sabem o motivo, a influência dos amigos e o prazer $(p<0,005)$.

$\mathrm{Na}$ avaliação dos adolescentes com o questionário DUSI, foi possível identificar o perfil da intensidade de problemas em relação ao uso de drogas. Deste modo, estabelecendo-se a 
densidade absoluta de problemas, a qual indicou a intensidade em cada área da vida do adolescente isoladamente (comportamento, estado de saúde, estado emocional, competência social, sistema familiar, situação escolar, situação de trabalho, relação interpessoal e lazer/recreação). Assim como a densidade relativa de problemas, que indicou a contribuição percentual de cada área no total de problemas.

A Tabela 2 ilustra a distribuição da amostra dos adolescentes segundo a intensidade de problemas.
Observa-se que o DUSI refletiu a intensidade de problemas em cada área isoladamente, além da densidade relativa, apresentou a contribuição percentual de cada área no total de problemas. As áreas com maiores índices de problemas que predispõem o uso de drogas pelos adolescentes foram: relação interpessoal, comportamento e lazer/recreação. Quanto à densidade global de problemas, os resultados apontaram novamente os maiores índices nas áreas de relação interpessoal e lazer/recreação. A escala de mentira não identificou questionários inválidos, portanto, todos os instrumentos de coleta dos dados foram preenchidos corretamente.

Tabela 2 - Descrição de problemas em relação ao uso de drogas por 480 adolescentes de escolas públicas, segundo o Drug Use Screening Inventory. Divinópolis, Minas Gerais, Brasil, 2014

\begin{tabular}{lccccccccc} 
& \multicolumn{3}{c}{ Densidade Absoluta de Problemas } & \multicolumn{3}{c}{ Densidade Relativa de Problemas } \\
\hline Áreas do DUSI & Mínimo & Máximo & Média & $\begin{array}{l}\text { Desvio } \\
\text { Padrão }\end{array}$ & Mínimo & Máximo & Média & $\begin{array}{c}\text { Desvio } \\
\text { Padrão }\end{array}$ \\
\hline Comportamento & 0 & 90,5 & 26,4 & 18,8 & 0 & 100 & 12,6 & 9,9 \\
\hline Estado de Saúde & 0 & 100 & 24,8 & 18,1 & 0 & 100 & 11,4 & 9,4 \\
\hline Estado Emocional & 0 & 81 & 23,8 & 18,1 & 0 & 50 & 10,1 & 6,1 \\
\hline Competência Social & 0 & 86,7 & 26,3 & 18,3 & 0 & 49 & 12,1 & 7,1 \\
\hline Sistema Familiar & 0 & 86,7 & 22,9 & 18,7 & 0 & 50 & 9,9 & 6,7 \\
\hline Situação Escolar & 0 & 81 & 23,1 & 16 & 0 & 100 & 11 & 7,1 \\
\hline Situação de Trabalho & 0 & 72,7 & 9,6 & 11 & 0 & 34,6 & 4,1 & 4,3 \\
\hline Relação Interpessoal & 0 & 93,3 & 25,1 & 19,7 & 0 & 51,7 & 11,1 & 7 \\
\hline Lazer Recreação & 0 & 130,8 & 28,8 & 18,8 & 0 & 56,5 & 13,7 & 8,3
\end{tabular}

\section{DISCUSSÃO}

Há evidências de que o uso de substâncias psicoativas é mais frequente entre o sexo masculino, superando em muito o feminino, embora não seja regra. A literatura aponta, ainda, que o uso de drogas consideradas "mais pesadas", como cocaína, cola e anfetamina, é mais frequente entre os homens ${ }^{(11-12)}$. Os achados constituem-se em desafios à saúde integral dos homens e servem de referencial para outros estudos que possam aprofundar a temática, incluindo a discussão de gênero.

A construção social do machismo muitas vezes estimula o menino a ter que comprovar sua coragem, momento em que jovens sem informação e condicionados ao padrão do macho, adotam comportamento nocivo à saúde. Em contrapartida, as meninas tendem a agir diferente dos meninos, ou seja, por serem consideradas socialmente como frágeis, são colocadas em uma posição de proteção, o que naturalmente as levam a evitar situações de risco nas quais sua saúde pode ser afetada ${ }^{(13-14)}$.

Contudo, estudo realizado com adolescentes na região sul do Brasil identificou a maior frequência no uso de tabaco pelo sexo feminino, enquanto o consumo de álcool foi maior no sexo masculino. Portanto, o consumo de tabaco eálcool é comum entre os adolescentes, independente do sexo. Estudos alertam que entre os consumidores de álcool foi evidenciado o uso de drogas ilícitas, o que demonstra o fator predisponente que o álcool exerce na indução ao uso de substâncias psicoativas ilícitas ${ }^{(13,15)}$.

É preocupante que a idade de início do uso de substâncias psicoativas esteja entre os 10 a 12 anos e que o principal motivo da busca seja por curiosidade, como constatado no presente estudo. Chamamos a atenção para a educação em saúde, como meio de propiciar a promoção da saúde mental, por meio da informação sobre os malefícios do uso e abuso do álcool e outras drogas. Vale ressaltar que os resultados 
coincidem com outros estudos, que também destacaram fatores motivadores para a imersão no mundo das drogas, como a necessidade de identificação com novos amigos e estruturas familiares instáveis, bem como a curiosidade em experimentar os efeitos, tanto do álcool como de drogas ilícitas ${ }^{(12,14,16)}$.

Pesquisa nacional( ${ }^{(8)}$ apontou que $55 \%$ dos adolescentes brasileiros já fizeram uso de álcool e $18,4 \%$ do tabaco, coincidindo com os achados deste estudo. De acordo com outra pesquisa ${ }^{(16)}$, o uso de substâncias psicoativas foi frequente entre o público adolescente na faixa etária dos 15 aos 19 anos, e mostrou-se relativamente elevado entre os adolescentes dos 10 aos 14 anos, desses constatou que $45,9 \%$ fizeram uso de álcool; $2,4 \%$ de maconha e $3,7 \%$ de outras drogas ilícitas, tais como cocaína, crack e ecstasy.

É válido destacar a responsabilidade das indústrias de substâncias psicoativas, como as de bebidas alcóolicas, que vêm utilizando cada vez mais da mídia e difusão artística para divulgar seus produtos. Esta presença quase que cotidiana nos espaços de comunicação e entretenimento, como as redes sociais virtuais e programas esportivos, torna o tema drogas bastante complexo de ser tratado. Pois, se por um lado são promovidos discursos que buscam construir uma imagem negativa da droga, por outro, há tantos outros que a constroem de forma positiva. A exemplo da mídia que veicula imagens extremamente favoráveis ao seu uso, utilizando-se de pessoas consideradas bonitas e bem sucedidas, na maioria das vezes artistas populares de renome, que promovem o uso relacionando-o à sociabilidade e à sexualidade.

No que tange ao consumo de drogas e a religião, apesar de neste estudo todos os adolescentes terem declarado seguir alguma prática religiosa, este aspecto não se evidenciou como um fator de proteção para o não uso de drogas. Assim, o fator protetivo da religião pode estar mais relacionado com o fato de aderir e participar efetivamente de uma religião, valorizar os ensinamentos religiosos, considerar importante crer em Deus e orar quando se está diante de alguma dificuldade, do que somente se sentir pertencente a alguma religião ${ }^{(10,17)}$.

Estudos $^{(9,12,15)}$ que referem à relação existente entre a religião e o uso de drogas, apontam maior consumo de substâncias psicoativas entre os estudantes com menor crença em Deus e menor frequência às práticas religiosas. Assim como sugerem que a religiosidade, expressa pela prática de uma crença, retarda o primeiro uso de drogas, também influenciando na menor frequência posterior do seu consumo.

No que se refere à raça, os resultados apontaram um maior número de adolescentes usuários de drogas que se declararam "amarelo/ oriental", seguidos pelos "negros", diferente dos resultados de outros estudos ${ }^{(14,16-17)}$, os quais apontaram os negros, seguidos pelos mulatos/ morenos como maiores usuários. Cabe ressaltar que as questões raciais estão ainda muito ligadas à herança cultural escravocrata e segregadora que permeou o processo de colonização do país. Isso resultou em parcela da população com baixo poder aquisitivo, como também foi comprovado neste estudo, com a maior parte dos adolescentes usuários de drogas pertencentes a famílias com renda mensal de até dois salários mínimos; residentes na periferia da cidade; em áreas desprovidas de infraestrutura (água tratada, rede de esgoto, ruas pavimentadas, serviços de transporte e lazer etc.) em exposição a situações de vulnerabilidade como violência, tráfico e consumo de drogas. Tais situações são o retrato fiel de vivências de riscos para o envolvimento com as drogas, somadas à gama de fatores que se conjugam com o ambiente $\mathrm{e}^{(12,14)}$.

Ao analisar a estrutura familiar, estudos realizados demonstram que a composição monoparental, ou seja, só a mãe ou o pai presente, predomina entre os adolescentes usuários de drogas e que esta estrutura familiar favorece o uso ${ }^{(12-13)}$. A família, por possuir o papel de inserir seus membros na sociedade e ser instituidora das relações primárias, influencia a forma como o adolescente reage à ampla oferta de droga atualmente. As relações familiares saudáveis desde o nascimento da criança servem como fator de proteção ao longo de toda a vida e, de forma muito particular, para o adolescente ${ }^{(17)}$.

No entanto, problemas enfrentados na adolescência, originados na infância, têm um contexto de realização muito mais ampliado. Sabe-se que as relações familiares representam um dos fatores mais relevantes a ser considerado, mas de forma combinada com outros. Estudos mostram que há relação linear entre o abuso de álcool pelos pais e de seus filhos, e sugerem que os padrões de comportamento dos pais e as interações familiares e, não só o fato deles beberem, são em boa parte responsáveis pelas atitudes dos filhos ${ }^{(17)}$.

$\mathrm{Na}$ adolescência, os jovens buscam uma "válvula de escape" para lidar com a ansiedade 
advinda pelas mais variadas fontes de conflitos e ambivalência. Há situações em que os pais não os tratam como crianças, com direitos de criança, mas também não os consideram adultos, com direitos de adultos. Assim, os adolescentes figuram numa posição intermediária em que seu papel não se encontra completamente definido, seja na família ou na sociedade ${ }^{(11-15)}$.

A literatura aponta que o uso de substâncias psicoativas está associado à ausência nas aulas e reprovação escolar. Ressalta-se que o uso de substâncias psicoativas, seja lícitas ou ilícitas, prejudica o rendimento escolar e podem levar ao abandono dos estudos ${ }^{(11-16)}$.

Deste modo, o compromisso com os estudos, a inserção no mercado de trabalho e o amparo familiar constituem-se como estratégias consideradas como de proteção ao adolescente para o não uso de drogas ${ }^{(16)}$. Neste estudo, a análise da situação escolar realmente revelou densidade de problemas baixa para o uso de drogas. Porém, é importante frisar que a repetência e o abandono escolar podem se constituir fatores de risco para o uso de drogas, ainda mais se a situação estiver atrelada à baixa autoestima.

Foi possível observar uma maior intensidade de problemas na área de lazer e recreação. Fato que indica a necessidade de políticas públicas voltadas para o jovem em termos de oferecer condições e atividades nesse âmbito, na perspectiva da atenção integral à saúde do adolescente.

A intensidade de problemas nas áreas de comportamento, competência social e relação interpessoal demonstram a gravidade da situação enfrentada por esses jovens, pois apresentaram uma relação direta existente entre a carência econômica, de acesso aos bens e serviços sociais com o uso de drogas.

\section{CONSIDERAÇÕES FINAIS}

O estudo identificou que há associação entre características sociodemográficas e o uso de álcool e outras drogas por adolescentes escolares, constituindo-se em desafio às ações intersetoriais das áreas de educação e saúde, na adoção de estratégias que visam tanto à prevenção como ao enfrentamento do uso e abuso de álcool e outras drogas, em especial na população de adolescentes, na perspectiva da promoção da saúde, através da sensibilização ao autocuidado. Assim, a educação em saúde constitui-se um meio para construção do conhecimento a respeito dos riscos e malefícios provocados por substâncias que levam à dependência química.

Conhecer as características sociodemográficas, bem como os fatores que levam ao uso do álcool e outras drogas por adolescentes, tem relevância no trabalho das equipes multiprofissionais de saúde, pois contribui no planejamento das intervenções pertinentes. A pesquisa, embora limitada pelo tamanho amostral, produziu conhecimento a respeito de um problema de saúde pública, cada vez mais frequente na sociedade, consistindo em motivação para outros estudos que possam corroborar com a saúde integral dos adolescentes.

Os resultados desse estudo revelaram que nem todas as localidades periféricas contam com ações de promoção da saúde, embora seja política pública nacional. Assim, recomenda-se ao setor público a efetivação da promoção da saúde integral dos adolescentes, em especial as atividades relacionadas com a redução da morbimortalidade em decorrência do uso abusivo do álcool e outras drogas, a prevenção e controle do tabagismo, a prevenção da violência e estímulo à cultura de paz, entre outras estratégias possíveis de serem implementadas.

\section{REFERÊNCIAS}

1. Silva CC, Costa MCO, de Carvalho RC, Amaral MT, Cruz CNL, da Silva MR. Initiation and consumption of psychoactive substances among adolescents and young adults in an anti-drug psychosocial care center. Ciên saúde coletiva. 2014; 19(3):737-45.

2. Mombelli MA, Marcon SS, Costa JB. Caracterização das internações psiquiátricas para desintoxicação de adolescentes dependentes químicos. Rev. bras. enferm. 2010; 5(63):735-40.

3. Lopes AP, Rezende MM. Consumo de substâncias psicoativas em estudantes do ensino médio. Psicol. teor. prat. 2014; 16(2):29-40.

4. Moreira A, Vóvio CL, de Micheli D. Drug abuse prevention in school: challenges and possibilities for the role of the educator. Educ. pesqui. 2015; 41(1):11934.

5. Nimtz MA, Tavares AMF, Maftum MA, Ferreira ACZ, Borba LO, Capistrano FC. Impacto do uso de drogas nos relacionamentos familiares de dependentes químicos. Cogitare enferm. 2014; 19(4):667-72.

6. Malbergier A, Cardoso LRD, Amaral RA, Santos VCV. Gender parity and drug use: are girls catching up with boys? Rev. Bras. Psiquiatr. 2012; 34(1):16-23.

7. Cardoso LRD, Malbergier A. Problemas escolares e o consumo de álcool e outras drogas entre adolescentes. 
Psicol. Esc. Educ. 2014; 18(1):27-34.

8. Malta DC, Oliveira-Campos M, do Prado RR, Andrade RR, de Mello SSC, Dias FCM, et al. Psychoactive substance use, family context and mental health among brazilian adolescents, national adolescent school-based health survey. Rev. bras. epidemiol. 2014; 17(1):46-61.

9. Silveira RE, Santos AS, Pereira GA. Consumo de álcool, tabaco e outras drogas entre adolescentes do ensino fundamental de um município brasileiro. Rev. Enf. Ref. 2014; 4(2):51-60.

10. Michelle D, Formigoni L. Screening of drug use in a teenage brazilian simple using the drug Use Screening Inventory (DUSI). Addictive Behaviors. 2000; 25(5):68391.

11. Michael E, Newcomb MB, Heather LC, Brian M. Sexual orientation, gender, and racial differences in illicit drug use in a sample of us high school students. Am J Public Health. 2014; 104(2):304-10.

12. Malta DC, Mascarenhas MD, Porto DL, Barreto SM, Morais Neto OL. Exposure to alcohol among adolescent students and associated factors. Rev Saude Publica. 2014; 48(1):52-62.

13. Brandão Neto W, Silva ARS, Almeida Filho AJ, de Lima LS, de Aquino JM, Monteiro EMLM. Intervenção educativa sobre violência com adolescentes: possibilidade para a enfermagem no contexto escolar. Esc. Anna Nery. 2014; 18(2):195-201.

14. Eaton DK, Kann L, Kinchen S, Whittle CD, Lim C, Wechsler $\mathrm{H}$. Youth risk behavior surveillance - United States, 2011. MMWR Surveill Summ. 2012; 61(4):1-162.

15. Valenca CN, Brandão ICA, Germano RM, de Vilar RLA, Monteiro Al. Abordagem da dependência de substancias psicoativas na adolescência: reflexão ética para a enfermagem. Esc. Anna Nery. 2013; 17(3):562-7.

16. Hadland SE, Harris SK. Youth marijuana use: state of the science for the practicing clinician. Curr Opin Pediatr. 2014; 26(4):420-7.

17. Barreto SM, Giatti L, Casado L, Moura L, Crespo C, Malta DC. Contextual factors associated with smoking among Brazilian adolescents. J Epidemiol Community Health. 2011; 66(8):723-9. 